\title{
Waterfilling Theorems in the Time-Frequency Plane for the Heat Channel and a Related Source
}

\author{
Edwin Hammerich \\ Ministry of Defence \\ Kulmbacher Str. 58-60, D-95030 Hof, Germany \\ E-mail: edwin.hammerich@ieee.org
}

\begin{abstract}
The capacity of the heat channel, a linear timevarying (LTV) filter with additive white Gaussian noise (AWGN), is characterized by waterfilling in the time-frequency plane. Similarly, the rate distortion function for a related nonstationary source is characterized by reverse waterfilling in the timefrequency plane. The source is formed by the white Gaussian noise response of the same LTV filter as before. The proofs of both waterfilling theorems rely on a specific Szegó theorem for a positive definite operator associated with the filter. An essentially self-contained proof of the Szegó theorem is given. The waterfilling theorems compare well with classical results of Gallager and Berger. In case of the nonstationary source it is observed that the part of the classical power spectral density (PSD) is taken by the Wigner-Ville spectrum (WVS).
\end{abstract}

\section{INTRODUCTION}

The characterization of the capacity of continuous-time (or waveform) channels by waterfilling in the frequency domain, going back to Shannon [1], has been given by Gallager [2] for linear time-invariant (LTI) waveform channels in great generality. At least since the advent of mobile communications there is a vivid interest in similar results for LTV channels; see [3], [4], [5] to cite only a few. Despite some progress-for instance LTV filter descriptions by pseudodifferential operators [6] based on the Weyl symbol [7], the spreading function [5] or others [4]-no (simple) characterizations of the capacity of LTV channels are known. The waterfilling theorems in the present paper may provide helpful examples in this connection (and beyond).

We consider the operator $\boldsymbol{P}_{\delta}^{(\gamma)}$ from the Hilbert space $L^{2}(\mathbb{R})$ of square-integrable functions $f: \mathbb{R} \rightarrow \mathbb{C} \cup\{\infty\}$ into itself given by

$$
\begin{aligned}
& \left(\boldsymbol{P}_{\delta}^{(\gamma)} f\right)(t)=e^{-\frac{t^{2}}{2 \alpha^{2}}} \\
& \cdot \frac{\beta}{\sqrt{2 \pi \cosh \delta}} \int_{-\infty}^{\infty} \exp \left[-\frac{\beta^{2}}{2}\left(\frac{t}{\cosh \delta}-t^{\prime}\right)^{2}\right] f\left(t^{\prime}\right) d t^{\prime},
\end{aligned}
$$

where $\alpha, \beta$ are any positive numbers satisfying $\alpha \beta>1$ and $\gamma, \delta>0$ are defined by $\gamma^{2}=\alpha / \beta, \operatorname{coth} \delta=\alpha \beta$ [8]. This operator, in its original form introduced as time-frequency localization operator in signal analysis [9], is deeply rooted in quantum mechanics (see [9], [10], [11] and the references therein); suffice it to say that the condition $\alpha \beta>1$ is a manifestation of the uncertainty principle. The operator (1) regarded as LTV filter for finite-energy signals $f(t)$ will play a central role in our investigations. Then, $t$ is time, $\alpha$ has the physical dimension of time and $\beta$ that of a frequency.

\section{The Heat Channel Revisited}

In contrast to [10], we define the heat channel as the continuous-time LTV channel

$$
\tilde{g}(t)=\left(\boldsymbol{P}_{\delta}^{(\gamma)} f\right)(t)+n(t),-\infty<t<\infty,
$$

where $\boldsymbol{P}_{\delta}^{(\gamma)}$ is the LTV filter (1), the real-valued filter input signals $f(t)$ are of finite energy and the noise signals $n(t)$ at the filter output are realizations of white Gaussian noise with two-sided PSD $N_{0} / 2=\theta^{2}>0$.

We now reduce the continuous-time heat channel to a (discrete) vector Gaussian channel following the approach in [2] for LTI waveform channels; our analysis is much simplified by the restriction to finite-energy input signals. Henceforth, we put $\rho=e^{-\delta}, \delta=\operatorname{arccoth}(\alpha \beta) \sim \frac{1}{\alpha \beta}(\alpha \beta \rightarrow \infty)$ [10]. For the LTV filter (1) we have the diagonalization [9], [10]

$$
\left(\boldsymbol{P}_{\delta}^{(\gamma)} f\right)(t)=\sum_{k=0}^{\infty} \rho^{k+\frac{1}{2}} a_{k}\left(D_{\gamma} H_{k}\right)(t)
$$

where $\left(D_{\gamma} H_{k}\right)(t)=\gamma^{-\frac{1}{2}} H_{k}(t / \gamma)$ is the dilated $k$ th Hermite function $H_{k}(t)$ and the coefficients are $a_{k}=\left\langle f, D_{\gamma} H_{k}\right\rangle$, $\left\langle f_{1}, f_{2}\right\rangle=\int_{-\infty}^{\infty} f_{1}(t) \overline{f_{2}(t)} d t$ denoting the inner product in $L^{2}(\mathbb{R}) .\left\{D_{\gamma} H_{k} ; k=0,1, \ldots\right\}$ forms a complete orthonormal system in $L^{2}(\mathbb{R})$. The perturbed filter output signal $g=\boldsymbol{P}_{\delta}^{(\gamma)} f, \tilde{g}(t)=g(t)+n(t)$, is passed through a bank of matched filters with impulse responses $h_{k}(t)=$ $\left(D_{\gamma} H_{k}\right)(-t), k=0,1, \ldots$ The matched filter output signals are sampled at time zero to yield $\left\langle\tilde{g}(t), h_{k}(-t)\right\rangle=b_{k}+n_{k}$, where $b_{k}=\left\langle g(t), h_{k}(-t)\right\rangle=\rho^{k+\frac{1}{2}} a_{k}$, and the detection errors $n_{k}=\left\langle n(t), h_{k}(-t)\right\rangle$ are realizations of independent identically distributed zero-mean Gaussian random variables $N_{k}$ with the variance $\theta^{2}, N_{k} \sim \mathcal{N}\left(0, \theta^{2}\right)$. From the detected values $\hat{b}_{k}=b_{k}+n_{k}$ we get the estimates $\hat{a}_{k}=\rho^{-k-\frac{1}{2}} \hat{b}_{k}=$ $a_{k}+z_{k}$ for the coefficients $a_{k}$ of the input signal $f$, where $z_{k}$ are realizations of independent Gaussian random variables $Z_{k} \sim \mathcal{N}\left(0, \theta^{2} \rho^{-2 k-1}\right)$. Thus, we are led to the infinitedimensional vector Gaussian channel

$$
Y_{k}=X_{k}+Z_{k}, k=0,1, \ldots
$$

where the noise $Z_{k}$ is distributed as described; it is the same channel as in [10, Def. 1]. Notice that the noise PSD $\theta^{2}$, measured in watts/Hz, has also the dimension of an energy. 
The interpretation of the ubiquitous time-frequency product $\alpha \beta$ as degrees of freedom (DoF) of filter output signals (cf. [10]) will be further substantiated in Section $\mathrm{V}-\mathrm{A}$

\section{A Specific SzEgő Theorem}

For a linear operator $\boldsymbol{A}: L^{2}(\mathbb{R}) \rightarrow L^{2}(\mathbb{R})$ the Weyl symbol $\sigma_{\boldsymbol{A}}(x, \xi)$ - when existing [6]—is defined by [7], [11]

$$
(\boldsymbol{A} f)(x)=\frac{1}{2 \pi} \iint_{\mathbb{R}^{2}} \sigma_{\boldsymbol{A}}\left(\frac{x+y}{2}, \xi\right) e^{i(x-y) \xi} f(y) d y d \xi
$$

The linear map $\boldsymbol{A} \mapsto \sigma_{\boldsymbol{A}}(x, \xi)$ (or its inverse) is called Weyl correspondence. For example, the positive definite operator $\boldsymbol{A}=\left(\boldsymbol{P}_{\delta}^{(\gamma)}\right)^{*} \boldsymbol{P}_{\delta}^{(\gamma)}=\boldsymbol{P}_{2 \delta}^{(\gamma)}$ has the Weyl symbol [8]

$$
\begin{aligned}
\sigma_{\boldsymbol{A}}(x, \xi) & =\frac{1}{\cosh \delta} e^{-(\tanh \delta)\left(\gamma^{-2} x^{2}+\gamma^{2} \xi^{2}\right)} \\
& =\frac{1}{\cosh \delta} \exp \left(-\frac{x^{2}}{\alpha^{2}}-\frac{\xi^{2}}{\beta^{2}}\right) .
\end{aligned}
$$

From now on, $\boldsymbol{A}$ will always stand for the foregoing operator and we shall write $\lambda_{k} \triangleq \rho^{2 k+1}, k=0,1, \ldots$, for its eigenvalues. The proof of the subsequent Szegó theorem, Thm. 1, is inspired by [11] although the Szegó theorems in [11] are inadequate for our purposes.

Definition 1: For any two functions $A, B:(1, \infty) \rightarrow \mathbb{R}$ the notation $A \doteq B$ means

$$
\lim _{x \rightarrow \infty} \frac{A(x)-B(x)}{x}=0,
$$

or, equivalently, $A(x)=B(x)+o(x)$ as $x \rightarrow \infty$, where $o(\cdot)$ denotes the standard Landau little-o symbol.

In our context, $x$ will always be $\alpha \beta$. Thus $A \doteq B$ implies that $A(\alpha \beta) /(\alpha \beta)=B(\alpha \beta) /(\alpha \beta)+\epsilon$ where $\epsilon \rightarrow 0$ as $\alpha \beta \rightarrow \infty$.

Lemma 1: For any polynomial $G_{N}(x, z)=\sum_{n=1}^{N} c_{n}(x) z^{n}$ with bounded variable coefficients $c_{n}(x) \in \mathbb{R}, x \in(1, \infty)$, it holds

$$
\sum_{k=0}^{\infty} G_{N}\left(\alpha \beta, \lambda_{k}\right) \doteq \frac{1}{2 \pi} \iint_{\mathbb{R}^{2}} G_{N}\left(\alpha \beta, \sigma_{\boldsymbol{A}}(x, \xi)\right) d x d \xi
$$

Proof: First, representation (3) (substitute $\delta$ by $2 \delta$ and $\rho$ by $\rho^{2}$ ) yields for any $f \in L^{2}(\mathbb{R})$ the expansion

$$
G_{N}(\alpha \beta, \boldsymbol{A}) f=\sum_{k=0}^{\infty} G_{N}\left(\alpha \beta, \lambda_{k}\right)\left\langle f, D_{\gamma} H_{k}\right\rangle D_{\gamma} H_{k} .
$$

Hence, operator $\boldsymbol{B} \triangleq G_{N}(\alpha \beta, \boldsymbol{A})$ has the trace

$$
\operatorname{tr} \boldsymbol{B}=\sum_{k=0}^{\infty} G_{N}\left(\alpha \beta, \lambda_{k}\right)
$$

the series being converging since $G_{N}(x, 0)=0 \forall x \in(1, \infty)$.

Second, we use the key observation [11, trace rule $(0.4)]$ to obtain (here and thereafter, double integrals extend over $\mathbb{R}^{2}$ )

$$
\operatorname{tr} \boldsymbol{B}=\frac{1}{2 \pi} \iint \sigma_{\boldsymbol{B}}(x, \xi) d x d \xi
$$

where $\sigma_{\boldsymbol{B}}(x, \xi)$ is the Weyl symbol of operator $\boldsymbol{B}$. By linearity of the Weyl correspondence, $\sigma_{\boldsymbol{B}}(x, \xi)$ has the expansion

$$
\sigma_{\boldsymbol{B}}(x, \xi)=\sum_{n=1}^{N} c_{n}(\alpha \beta) \sigma_{\boldsymbol{A}^{n}}(x, \xi) .
$$

Since for any $\gamma>0$ held constant the family of operators $\left\{\boldsymbol{P}_{\delta}^{(\gamma)} ; \delta>0\right\}$ forms a semigroup with respect to $\delta$ (see [8]), it follows that $\boldsymbol{A}^{n}=\boldsymbol{P}_{2 n \delta}^{(\gamma)}$. In Eq. (5), substitute operator $\boldsymbol{A}$ by $\boldsymbol{A}^{n}$ and parameter $\delta$ by $n \delta$. Because of $\tanh (n \delta)=$ $(n \tanh \delta)(1+o(1))$ we then obtain

$$
\begin{aligned}
\sigma_{\boldsymbol{A}^{n}}(x, \xi)= & \frac{1}{\cosh (n \delta)} e^{-\tanh (n \delta)\left(\gamma^{-2} x^{2}+\gamma^{2} \xi^{2}\right)} \\
= & (1+o(1))\left(\sigma_{\boldsymbol{A}}(x, \xi)\right)^{n} \\
& \cdot \exp \left[-o(1)\left(\frac{x^{2}}{\alpha^{2}}+\frac{\xi^{2}}{\beta^{2}}\right)\right],
\end{aligned}
$$

where the Landau symbol $o(1)$ stands for various quantities vanishing as $\delta \rightarrow 0$ (or $\alpha \beta \rightarrow \infty$ ). We now estimate

$$
\begin{aligned}
\operatorname{tr} \boldsymbol{B} & =\frac{1}{2 \pi} \iint \sigma_{\boldsymbol{B}}(x, \xi) d x d \xi \\
& =\left[\frac{1}{2 \pi} \iint G_{N}\left(\alpha \beta, \sigma_{\boldsymbol{A}}\left(\alpha x^{\prime}, \beta \xi^{\prime}\right)\right) d x^{\prime} d \xi^{\prime}+\epsilon\right] \alpha \beta \\
& =\frac{1}{2 \pi} \iint G_{N}\left(\alpha \beta, \sigma_{\boldsymbol{A}}(x, \xi)\right) d x d \xi+\epsilon \alpha \beta
\end{aligned}
$$

where $\epsilon \rightarrow 0$ as $\alpha \beta \rightarrow \infty$. Eq. (8) in combination with Eq. (7) concludes the proof.

Theorem 1 (Szegó Theorem): Let $g:[0, \Delta] \rightarrow \mathbb{R}, \Delta \in$ $(0, \infty)$, be a continuous function such that $\lim _{x \rightarrow 0+} g(x) / x$ exists. For any functions $a, b:(1, \infty) \rightarrow \mathbb{R}$, where $a(x)$ is bounded and $b(x) \in[0, \Delta]$, define the function $G(x, z)=$ $a(x) g(b(x) z),(x, z) \in(1, \infty) \times[0,1]$. Then it holds

$$
\sum_{k=0}^{\infty} G\left(\alpha \beta, \lambda_{k}\right) \doteq \frac{1}{2 \pi} \iint_{\mathbb{R}^{2}} G\left(\alpha \beta, \sigma_{\boldsymbol{A}}(x, \xi)\right) d x d \xi .
$$

Proof: The function $f(x)=g(x) / x, x \in(0, \Delta]$, has a continuous extension $F(x)$ onto the compact interval $[0, \Delta]$. By virtue of the Weierstrass approximation theorem, for any $m \in \mathbb{N}$ there exists a polynomial $F_{N_{m}-1}(x)$ of some degree $N_{m}-1$ such that $\left|F(x)-F_{N_{m}-1}(x)\right| \leq \epsilon_{m}=\frac{1}{m}$ for all $x \in$ $[0, \Delta]$. Consequently, the polynomial $g_{N_{m}}(x)=x F_{N_{m}-1}(x)$ of degree $N_{m}$ satisfies the inequality

$$
\left|g(x)-g_{N_{m}}(x)\right| \leq \epsilon_{m} x, x \in[0, \Delta] .
$$

Define the polynomial with variable coefficients $G_{N_{m}}(x, z)=a(x) g_{N_{m}}(b(x) z)$. We now show that

$$
(\alpha \beta)^{-1} \sum_{k=0}^{\infty} G_{N_{m}}\left(\alpha \beta, \lambda_{k}\right) \rightarrow(\alpha \beta)^{-1} \sum_{k=0}^{\infty} G\left(\alpha \beta, \lambda_{k}\right)
$$

and

$$
\begin{aligned}
& \frac{(\alpha \beta)^{-1}}{2 \pi} \iint_{\mathbb{R}^{2}} G_{N_{m}}\left(\alpha \beta, \sigma_{\boldsymbol{A}}(x, \xi)\right) d x d \xi \\
& \rightarrow \frac{(\alpha \beta)^{-1}}{2 \pi} \iint_{\mathbb{R}^{2}} G\left(\alpha \beta, \sigma_{\boldsymbol{A}}(x, \xi)\right) d x d \xi
\end{aligned}
$$


as $m \rightarrow \infty$, uniformly for all $\alpha \beta \in(1, \infty)$.

Proof of (11): By Ineq. (10) we get

$$
\begin{aligned}
& \left|\sum_{k=0}^{\infty} G\left(\alpha \beta, \lambda_{k}\right)-\sum_{k=0}^{\infty} G_{N_{m}}\left(\alpha \beta, \lambda_{k}\right)\right| \\
& \quad \leq \sum_{k=0}^{\infty}\left|G\left(\alpha \beta, \lambda_{k}\right)-G_{N_{m}}\left(\alpha \beta, \lambda_{k}\right)\right| \\
& \quad \leq M \epsilon_{m} \Delta \sum_{k=0}^{\infty} \lambda_{k},
\end{aligned}
$$

where $M=\sup \{|a(x)| ; x>1\}<\infty$ and $\sum_{k=0}^{\infty} \lambda_{k}=\rho /(1-$ $\left.\rho^{2}\right)=\alpha \beta /(2 \cosh \delta)<\alpha \beta / 2, \alpha \beta>1$. After devision of the inequalitiy by $\alpha \beta$, convergence in (11) follows as claimed.

Proof of (12): Similarly,

$$
\begin{aligned}
& \mid \iint G\left(\alpha \beta, \sigma_{\boldsymbol{A}}(x, \xi)\right) d x d \xi \\
& \quad-\iint G_{N_{m}}\left(\alpha \beta, \sigma_{\boldsymbol{A}}(x, \xi)\right) d x d \xi \mid \\
& \quad \leq \quad M \epsilon_{m} \Delta \iint \sigma_{\boldsymbol{A}}(x, \xi) d x d \xi .
\end{aligned}
$$

Since $(2 \pi)^{-1} \iint \sigma_{\boldsymbol{A}}(x, \xi) d x d \xi=\alpha \beta /(2 \cosh \delta)$, after division by $2 \pi \alpha \beta$ we come to the same conclusion as before.

Now, choose a (large) number $m \in \mathbb{N}$, so that the left-hand sides in (11), (12) become arbitrarily close to their respective limit. Replace function $G$ in Eq. (9) with the polynomial $G_{N_{m}}$. Then, by Lem. 11 and the uniform convergence in (11), (12), the theorem follows.

\section{WATERFILling THEOREM FOR THE CAPACITY OF THE HEAT CHANNEL}

The function $N(t, \omega)$ occurring in the next theorem is defined as

$$
N(t, \omega)=\frac{\theta^{2}}{2 \pi} \cdot(\cosh \delta) \exp \left(\frac{t^{2}}{\alpha^{2}}+\frac{\omega^{2}}{\beta^{2}}\right) .
$$

$O(\cdot)$ denotes the standard Landau big-O symbol and $x^{+}$the positive part of $x \in \mathbb{R}, x^{+}=\max \{0, x\}$.

Theorem 2: Assume that the average energy $S$ of the input signal depends on $\alpha \beta$ such that $S(\alpha \beta)=O(\alpha \beta)$ as $\alpha \beta \rightarrow \infty$. Then for the capacity (in nats per transmission) of the heat channel it holds

$$
C \doteq \frac{1}{2 \pi} \iint_{\mathbb{R}^{2}} \frac{1}{2} \ln \left(1+\frac{(\nu-N(t, \omega))^{+}}{N(t, \omega)}\right) d t d \omega,
$$

where $\nu$ is chosen so that

$$
S \doteq \iint_{\mathbb{R}^{2}}(\nu-N(t, \omega))^{+} d t d \omega
$$

Proof: The first part of the proof is accomplished by waterfilling on the noise variances [2, Thm. 7.5.1] (as in the proof of [10, Thm. 1]). Let $\nu_{k}^{2}=\theta^{2} \rho^{-2 k-1}=\theta^{2} \lambda_{k}^{-1}, k=0,1, \ldots$, be the noise variance in the $k$ th subchannel of the discretized heat channel (4). The "water level" $\sigma^{2}>\nu_{0}^{2}$ (excluding the trivial case $S=0$ ) is defined by the condition

$$
S=\sum_{k=0}^{\infty}\left(\sigma^{2}-\nu_{k}^{2}\right)^{+}=\sum_{k=0}^{K-1}\left(\sigma^{2}-\nu_{k}^{2}\right)
$$

where $K=\max \left\{k \in \mathbb{N} ; \nu_{k-1}^{2}<\sigma^{2}\right\}$ is the number of subchannels in the resulting finite-dimensional vector Gaussian channel. The capacity $C$ of that vector channel is achieved when the components $X_{k}$ of the input vector $\left(X_{0}, \ldots, X_{K-1}\right)$ are independent random variables $\sim \mathcal{N}\left(0, \sigma^{2}-\nu_{k}^{2}\right)$; then

$$
C=\sum_{k=0}^{K-1} \frac{1}{2} \ln \left(1+\frac{\sigma^{2}-\nu_{k}^{2}}{\nu_{k}^{2}}\right) \quad \text { nats. }
$$

In the second part of the proof we apply the above Szegó theorem, Thm. 1. To start with, note that $\sigma^{2}$ is dependent on $\alpha \beta$ and that always $\sigma^{2}=\sigma^{2}(\alpha \beta)>\theta^{2}$. On the other hand, the function $\sigma^{2}(\alpha \beta)$ is finitely upper bounded as $\alpha \beta \rightarrow \infty$ because of the growth condition imposed on $S=S(\alpha \beta)$ and the equation (refer to the proof of [10, Thm. 1])

$$
S \doteq \frac{\alpha \beta}{2} \theta^{2} \cdot\left(\frac{\sigma^{2}}{\theta^{2}} \ln \frac{\sigma^{2}}{\theta^{2}}-\frac{\sigma^{2}}{\theta^{2}}+1\right),
$$

observing that the function $y=x \ln x-x+1$ is positive and convex downward for $x>1$. Define

$$
\ln _{+} x=\left\{\begin{array}{cl}
\max \{0, \ln x\} & \text { if } x>0 \\
0 & \text { if } x=0 .
\end{array}\right.
$$

By Eq. (17) we have

$$
\begin{aligned}
C & =\sum_{k=0}^{\infty} \frac{1}{2} \ln _{+}\left(\frac{\sigma^{2}(\alpha \beta)}{\theta^{2}} \lambda_{k}\right) \\
& =\sum_{k=0}^{\infty} a(\alpha \beta) g\left(b(\alpha \beta) \lambda_{k}\right),
\end{aligned}
$$

where $a(\alpha \beta)=1, b(\alpha \beta)=\sigma^{2}(\alpha \beta) / \theta^{2}, g(x)=\frac{1}{2} \ln _{+} x, x \in$ $[0, \Delta]$, and $\Delta$ is chosen so that $b(\alpha \beta) \leq \Delta<\infty$ when $\alpha \beta$ is large enough. The latter choice is possible since $\sigma^{2}(\alpha \beta)$ remains bounded as $\alpha \beta \rightarrow \infty$; without loss of generality (w.l.o.g.), we assume $b(\alpha \beta) \in[0, \Delta]$ for all $\alpha \beta \in(1, \infty)$. Then, by Thm. 1 it follows that $C=C(\alpha \beta)$ satisfies

$$
\begin{aligned}
C & \doteq \frac{1}{2 \pi} \iint \frac{1}{2} \ln _{+}\left(\frac{\sigma^{2}(\alpha \beta)}{\theta^{2}} \sigma_{\boldsymbol{A}}(x, \xi)\right) d x d \xi \\
& =\frac{1}{2 \pi} \iint \frac{1}{2} \ln \left[1+\frac{\left(\frac{\sigma^{2}(\alpha \beta)}{2 \pi}-N(t, \omega)\right)^{+}}{N(t, \omega)}\right] d t d \omega
\end{aligned}
$$

where $N(t, \omega) \triangleq \frac{\theta^{2}}{2 \pi} \sigma_{\boldsymbol{A}}(t, \omega)^{-1}$. Next, rewrite Eq. (16) as

$$
S=\sum_{k=0}^{\infty} \sigma^{2}(\alpha \beta)\left(1-\frac{1}{\frac{\sigma^{2}(\alpha \beta)}{\theta^{2}} \lambda_{k}}\right)^{+} .
$$

Put $a(\alpha \beta)=\sigma^{2}(\alpha \beta), b(\alpha \beta)=\sigma^{2}(\alpha \beta) / \theta^{2}$ and define

$$
g(x)=\left\{\begin{array}{cl}
\left(1-\frac{1}{x}\right)^{+} & \text {if } x>0 \\
0 & \text { if } x=0 .
\end{array}\right.
$$


Again, w.l.o.g., we may assume that $a(\alpha \beta)$ is bounded and $b(\alpha \beta) \in[0, \Delta]$ for all $\alpha \beta \in(1, \infty)$ where $\Delta$ is chosen as above. Then, by Thm. 1 it follows that

$$
\begin{aligned}
S & \doteq \frac{1}{2 \pi} \iint \sigma^{2}(\alpha \beta)\left(1-\frac{1}{\frac{\sigma^{2}(\alpha \beta)}{\theta^{2}} \sigma_{\boldsymbol{A}}(x, \xi)}\right)^{+} d x d \xi \\
& =\iint\left(\frac{\sigma^{2}(\alpha \beta)}{2 \pi}-N(t, \omega)\right)^{+} d t d \omega .
\end{aligned}
$$

Finally, replacement of $\frac{\sigma^{2}(\alpha \beta)}{2 \pi}$ by the parameter $\nu$ completes the proof.

Remark 1: Note that the use of Landau symbols does not mean that we need to pass to the limit (here as $\alpha \beta \rightarrow \infty$ ). Rather, the dotted equations (14), (15) may give useful approximations even when $\alpha \beta$ is finite (but large enough).

For example, if $\alpha \beta=50$ and $S=20, \theta^{2}=0.01$ (units omitted), then Eq. (14) yields with $\nu=0.051$ an approximate capacity of 75.1043 nats/transmission-the exact one (determined numerically) is 75.1017. For that $\nu$, the integral on the right-hand side of Eq. (15) evaluates to 20.0013 - a value close to $S$. Actually, $\nu$ has been computed as described in the above proof.

Eqs. (14), (15) may also be taken for a parametric representation of the function $C=C(S)$ (neglecting the error terms). To get rid of the latter one might prefer to average with respect to the $\operatorname{DoF} \alpha \beta$ and letting $\alpha \beta \rightarrow \infty$.

When $\beta$ is held constant and $\alpha \rightarrow \infty$, the LTV channel (2) appears to tend towards an LTI waveform channel according to Gallager's model in [2, Ch. 8] with LTI filter with impulse response $h_{1}(t)=(\beta / \sqrt{2 \pi}) \exp \left(-\beta^{2} t^{2} / 2\right)$ (we stick to the notations in [2]). It is therefore interesting to compare Thm. 22 with Gallager's capacity theorem [2, Thm. 8.5.1] when applied to that particular waveform channel (with AWGN of noise PSD $N_{0} / 2=\theta^{2}$ ). By Gallager's theorem we obtain for the capacity $C$ (in bits per second) and average input power $S$ the parametric representation

$$
\begin{aligned}
C & =\frac{1}{2 \pi} \int_{-\infty}^{\infty} \frac{1}{2} \log _{2}\left(1+\frac{\left(\nu-N_{1}(\omega)\right)^{+}}{N_{1}(\omega)}\right) d \omega \\
S & =\int_{-\infty}^{\infty}\left(\nu-N_{1}(\omega)\right)^{+} d \omega,
\end{aligned}
$$

where $\nu$ is the parameter, $\omega$ is angular frequency, and

$$
N_{1}(\omega)=\frac{\theta^{2}}{2 \pi} \cdot \exp \left(\frac{\omega^{2}}{\beta^{2}}\right) \text {. }
$$

We observe perfect formal analogy between the waterfilling formulas (19), (20) and those in Thm. 2. Moreover, $N(t, \omega)$ in (13) tends to $N_{1}(\omega)$ as $\alpha \rightarrow \infty$ for any $t, \omega$ held constant.

\section{REVERSE WATERFILLING THEOREM FOR A RELATED NONSTATIONARY SOURCE}

In the present section we consider the nonstationary source formed by the nonstationary zero-mean Gaussian process given by the Karhunen-Loève expansion

$$
X(t)=\sum_{k=0}^{\infty} X_{k}\left(D_{\gamma} H_{k}\right)(t), t \in \mathbb{R},
$$

where the coefficients $X_{k}, k=0,1, \ldots$, are independent random variables $\sim \mathcal{N}\left(0, \sigma_{k}^{2}\right)$ with the variances $\sigma_{k}^{2}=$ $\sigma^{2} \rho^{2 k+1}=\sigma^{2} \lambda_{k}, \sigma>0$. It is the response of the LTV filter (1) on white Gaussian noise with PSD $N_{0} / 2=\sigma^{2}$; cf. [12].

\section{A. Wigner-Ville Spectrum of the Source}

The WVS $\Phi(t, \omega)$ of the nonstationary random process $\{X(t), t \in \mathbb{R}\}$ in (21) describes its density of (average) energy in the time-frequency plane [13]. The WVS may be regarded as the nonstationary counterpart to the PSD of a stationary random process. It is defined by means of the Wigner transform [6] of the realizations $x(t)$ of $\{X(t)\}$ and then taking the expectation (for details refer to [12]). Then $\Phi(t, \omega)=(2 \pi)^{-1} \int e^{-i \omega t^{\prime}} r\left(t+t^{\prime} / 2, t-t^{\prime} / 2\right) d t^{\prime}$, where $r\left(t_{1}, t_{2}\right)=\mathbb{E}\left[X\left(t_{1}\right) \overline{X\left(t_{2}\right)}\right]$ is the autocorrelation function; in our case we obtain [12]

$$
\Phi(t, \omega)=\frac{\sigma^{2}}{2 \pi} \cdot \frac{1}{\cosh \delta} \exp \left(-\frac{t^{2}}{\alpha^{2}}-\frac{\omega^{2}}{\beta^{2}}\right) .
$$

Indeed, the average energy $E=E(\alpha \beta)$ of the process (21) is

$$
E=\sum_{k=0}^{\infty} \sigma_{k}^{2}=\iint_{\mathbb{R}^{2}} \Phi(t, \omega) d t d \omega\left(\doteq \frac{\alpha \beta}{2} \sigma^{2}\right) .
$$

Notice that the WVS (22) is proportional to the Weyl symbol (6) (with $x \leftarrow t, \xi \leftarrow \omega$ ). Consequently, it defines the same ellipse of concentration as described in [10].

\section{B. $R(D)$ by Reverse Waterfilling in the Time-Frequency Plane}

Substitute the continuous-time Gaussian process $\{X(t), t \in$ $\mathbb{R}\}$ in $(21)$ by the sequence of coefficient random variables $\boldsymbol{X}=X_{0}, X_{1}, \ldots$ For an estimate $\hat{\boldsymbol{X}}=\hat{X}_{0}, \hat{X}_{1}, \ldots$ of $\boldsymbol{X}$ we take the squared-error distortion $D=\mathbb{E}\left[\sum_{k=0}^{\infty}\left(X_{k}-\hat{X}_{k}\right)^{2}\right]$ as distortion measure.

The Landau symbol $\Omega(\cdot)$ occurring in the next theorem is defined for any two functions as in Def. 1 as follows: $A(x)=\Omega(B(x))$ as $x \rightarrow \infty$ if $B(x)>0$ and $\liminf _{x \rightarrow \infty} A(x) / B(x)>0$.

Theorem 3: Assume that the foregoing average distortion $D$ depends on $\alpha \beta$ such that $D(\alpha \beta)=\Omega(\alpha \beta)$ as $\alpha \beta \rightarrow \infty$. Then the rate distortion function $R=R(D)$ for the nonstationary source (21) is given by

$$
R \doteq \frac{1}{2 \pi} \iint_{\mathbb{R}^{2}} \max \left\{0, \frac{1}{2} \ln \frac{\Phi(t, \omega)}{\lambda}\right\} d t d \omega,
$$

where $\lambda$ is chosen so that

$$
D \doteq \iint_{\mathbb{R}^{2}} \min \{\lambda, \Phi(t, \omega)\} d t d \omega .
$$

The rate is measured in nats per realization of the source.

Proof: First, assume $0<D<E$ where $E$ is the average energy (23). The reverse waterfilling argument for a finite number of independent Gaussian sources [15], [14] carries over to our case without changes resulting in a finite collection of Gaussian sources $X_{0}, \ldots, X_{K-1}$ where $K=\max \{k \in$ 
$\left.\mathbb{N} ; \sigma_{k-1}^{2}>\theta^{2}\right\}$ and the "(ground-)water table" $\theta^{2}>0$ is determined by the condition

$$
D=\sum_{k=0}^{\infty} \min \left\{\theta^{2}, \sigma_{k}^{2}\right\} .
$$

Then $K \geq 1$ and the rate distortion function $R=R(D)$ for the parallel Gaussian source $\left(X_{0}, \ldots, X_{K-1}\right)$ is given by [14 Thm. 10.3.3]

$$
R=\sum_{k=0}^{K-1} \frac{1}{2} \ln \frac{\sigma_{k}^{2}}{\theta^{2}} \quad \text { nats. }
$$

Now we apply the above Szegô theorem, Thm. 1. Note first that $\theta^{2}$ is dependent on $\alpha \beta$ and that always $\theta^{2}=\theta^{2}(\alpha \beta)<\sigma^{2}$. On the other hand, the function $\theta^{2}(\alpha \beta)$ is positively lower bounded as $\alpha \beta \rightarrow \infty$ because of the growth condition imposed on $D=D(\alpha \beta)$ and the equation (refer to the proof of [10. Thm. 2])

$$
D \doteq \frac{\alpha \beta}{2} \sigma^{2} \cdot\left(\frac{\theta^{2}}{\sigma^{2}}-\frac{\theta^{2}}{\sigma^{2}} \ln \frac{\theta^{2}}{\sigma^{2}}\right),
$$

observing that the function $y=x-x \ln x$ is positive and strictly monotonically increasing for $0<x \leq 1$ and $y \rightarrow 0$ as $x \rightarrow 0+$. By Eq. 26) we have,

$$
\begin{aligned}
D & =\sum_{k=0}^{\infty} \theta^{2}(\alpha \beta) \min \left\{1, \frac{\sigma^{2}}{\theta^{2}(\alpha \beta)} \lambda_{k}\right\} \\
& =\sum_{k=0}^{\infty} a(\alpha \beta) g\left(b(\alpha \beta) \lambda_{k}\right),
\end{aligned}
$$

where $a(\alpha \beta)=\theta^{2}(\alpha \beta), b(\alpha \beta)=\sigma^{2} / \theta^{2}(\alpha \beta), g(x)=$ $\min \{1, x\}, x \in[0, \Delta]$, and $\Delta$ is chosen so that $b(\alpha \beta) \leq \Delta<$ $\infty$ when $\alpha \beta$ is large enough. The latter choice is possible since $\theta^{2}(\alpha \beta)$ remains positively lower bounded as $\alpha \beta \rightarrow \infty$; w.l.o.g. we assume here and thereafter that $b(\alpha \beta) \in[0, \Delta]$ for all $\alpha \beta \in(1, \infty)$. $a(\alpha \beta)$ is always bounded. Therefore, by Thm. 11 we infer

$$
\begin{aligned}
D & \doteq \frac{1}{2 \pi} \iint \theta^{2}(\alpha \beta) \min \left\{1, \frac{\sigma^{2}}{\theta^{2}(\alpha \beta)} \sigma_{\boldsymbol{A}}(x, \xi)\right\} d x d \xi \\
& =\iint \min \left\{\frac{\theta^{2}(\alpha \beta)}{2 \pi}, \Phi(t, \omega)\right\} d t d \omega,
\end{aligned}
$$

where $\Phi(t, \omega)=\frac{\sigma^{2}}{2 \pi} \sigma_{\boldsymbol{A}}(t, \omega)$ is the WVS (22) of the source. Next, rewrite Eq. (27) as

$$
R=\sum_{k=0}^{\infty} \frac{1}{2} \ln _{+}\left(\frac{\sigma^{2}}{\theta^{2}(\alpha \beta)} \lambda_{k}\right),
$$

where $\ln _{+}$is as defined in (18). Taking $a(\alpha \beta)=1, b(\alpha \beta)=$ $\sigma^{2} / \theta^{2}(\alpha \beta), g(x)=\frac{1}{2} \ln _{+} x, x \in[0, \Delta], \Delta$ chosen as before, by Thm. 1 it follows that

$$
\begin{aligned}
R & \doteq \frac{1}{2 \pi} \iint \frac{1}{2} \ln _{+}\left(\frac{\sigma^{2}}{\theta^{2}(\alpha \beta)} \sigma_{\boldsymbol{A}}(x, \xi)\right) d x d \xi \\
& =\frac{1}{2 \pi} \iint \frac{1}{2} \ln _{+}\left[\frac{\Phi(t, \omega)}{\frac{\theta^{2}(\alpha \beta)}{2 \pi}}\right] d t d \omega .
\end{aligned}
$$

Finally, replacement of $\frac{\theta^{2}(\alpha \beta)}{2 \pi}$ by the parameter $\lambda$ concludes the proof in case $0<D<E$.

When $D=E$ (the case $D=0$ is precluded by asssumption as $\alpha \beta \rightarrow \infty$; the case $D>E$ is of no interest) then, as always, $R(D)=0$. Choosing in the theorem the parameter $\lambda=\Phi(0,0)=\max _{t, \omega} \Phi(t, \omega)$, we obtain correctly $R \doteq 0, D \doteq E$. This completes the proof of the theorem.

As in Section IV] Eqs. (24), (25) may also be taken for a parametric representation of the $R(D)$ function. In parametric form, the $R(D)$ function has been given by Berger [15] for a broad class of stationary random processes. In the latter parametric interpretation, Eq. (24) is in perfect analogy to [15, Eq. (4.5.52)] (with WVS instead of PSD), likewise Eq. 25 with regard to [15, Eq. (4.5.51)] (apart from a factor $\frac{1}{2 \pi}$ ).

\section{CONCLUSION}

Two waterfilling theorems in the time-frequency plane were stated in terms of the Weyl symbol (or its reciprocal) and rigorous proofs have been given. The relevance of the Weyl symbol was reflected by its equivalence with the WVS of the nonstationary source. The proof of a specific Szegó theorem took advantage of the semigroup property of the LTV filter. Although the latter feature is not necessarily a prerequisite for a conceivable generalization of the present results, asymptotic analysis and notation seem unavoidable.

\section{REFERENCES}

[1] C. E. Shannon, "Communication in the presence of noise," Proc. IRE, vol. 37 , pp. 10-21, 1949.

[2] R. G. Gallager, Information Theory and Reliable Communication. New York, NY: Wiley, 1968.

[3] S. Barbarossa and A. Scaglione, "On the capacity of linear time-varying channels," Proc. IEEE Int. Conf. Acustics Speech Signal Process., 1999, vol. 5, pp. 2627-2630.

[4] P. Jung, "On the Szegö-asymptotics for doubly-dispersive Gaussian channels," Proc. IEEE Int. Symp. Information Theory, St. Petersburg, Russia, 2011, pp. 2852-2856.

[5] B. Farrell and T. Strohmer, "Eigenvalue estimates and mutual information for the linear time-varying channel," IEEE Trans. Inf. Theory, vol. 57, pp. 5710-5718, 2011.

[6] K. Gröchenig, Foundations of Time-Frequency Analysis. Boston: Birkhäuser, 2001.

[7] W. Kozek and F. Hlawatsch, "Time-frequency representation of linear time-varying systems using the Weyl symbol," IEE Sixth Int. Conf. on Digital Signal Process. in Communications, Loughborough, UK, 1991, pp. 25-30.

[8] E. Hammerich, "A sampling theorem for time-frequency localized signals," Sampl. Theory Signal Image Process., vol. 3, pp. 45-81, 2004.

[9] I. Daubechies, "Time-frequency localization operators: A geometric phase space approach," IEEE Trans. Inf. Theory, vol. 34, pp. 605-612, 1988.

[10] E. Hammerich, "On the heat channel and its capacity," Proc. IEEE Int. Symp. Information Theory, Seoul, Korea, 2009, pp. 1809-1813.

[11] A. J. E. M. Janssen and S. Zelditch, "Szegö limit theorems for the harmonic oscillator," Trans. Amer. Math. Soc., vol. 280, pp. 563-587, 1983.

[12] E. Hammerich, "On the capacity of the heat channel, waterfilling in the time-frequency plane, and a C-NODE relationship," 2014 [Online]. Available: arXiv: $1101.0287 \mathrm{v} 4$

[13] P. Flandrin and W. Martin, "The Wigner-Ville spectrum of nonstationary random signals," in The Wigner Distribution, W. Mecklenbräuker and F. Hlawatsch, Eds. Amsterdam: Elsevier, 1997, pp. 211-267.

[14] T. M. Cover and J. A. Thomas, Elements of Information Theory. 2nd ed. Hoboken, NJ: Wiley, 2006.

[15] T. Berger, Rate Distortion Theory: A Mathematical Basis for Data Compression. Englewood Cliffs, NJ: Prentice-Hall, 1971. 